University of Wollongong

Research Online

Faculty of Engineering and Information

Faculty of Engineering and Information

Sciences - Papers: Part A

Sciences

$1-1-2014$

\title{
Spatial shift unwrapping for digital fringe profilometry based on spatial shift estimation
}

Pu Cao

University of Wollongong, pc241@uowmail.edu.au

Jiangtao Xi

University of Wollongong, jiangtao@uow.edu.au

Yanguang Yu

University of Wollongong, yanguang@uow.edu.au

Qinghua Guo

University of Wollongong, qguo@uow.edu.au

Follow this and additional works at: https://ro.uow.edu.au/eispapers

Part of the Engineering Commons, and the Science and Technology Studies Commons

Research Online is the open access institutional repository for the University of Wollongong. For further information contact the UOW Library: research-pubs@uow.edu.au 


\title{
Spatial shift unwrapping for digital fringe profilometry based on spatial shift estimation
}

\begin{abstract}
An approach is presented to solve the problem of spatial shift wrapping associated with spatial shift estimation-based fringe pattern profilometry (FPP). This problem arises as the result of fringe reuses (that is, use of fringes with periodic light intensity variance), and the spatial shift can only be identified without ambiguity within the range of a fringe width. It is demonstrated that the problem is similar to the phase unwrapping problem associated with the phase-detection-based FPP, and the proposed method is inspired by the existing ideas of using multiple images with different wavelengths proposed for phase unwrapping. The effectiveness of the proposed method is verified by comparing experimental results against several objects, with the last object consisting of more complex surface features. We conclude by showing that our method is successful in reconstructing the fine details of the more complex object.
\end{abstract}

\section{Keywords}

fringe pattern profilometry, 3-D measurement, phase unwrapping

\section{Disciplines}

Engineering | Science and Technology Studies

\section{Publication Details}

P. Cao, J. Xi, Y. Yu \& Q. Guo, "Spatial shift unwrapping for digital fringe profilometry based on spatial shift estimation," Journal of Electronic Imaging, vol. 23, (4) pp. 043002-1 - 043002-9, 2014. 


\section{Electronnic Imaging}

JElectroniclmaging.org

\section{Spatial shift unwrapping for digital fringe profilometry based on spatial shift estimation}

$\mathrm{Pu} \mathrm{Cao}$

Jiangtao Xi

Yanguang Yu

Qinghua Guo 


\title{
Spatial shift unwrapping for digital fringe profilometry based on spatial shift estimation
}

\author{
Pu Cao, Jiangtao Xi, ${ }^{*}$ Yanguang Yu, and Qinghua Guo \\ University of Wollongong, School of Electrical, Computer and Telecommunications Engineering, Northfields Avenue, \\ Wollongong NSW2522, Australia
}

\begin{abstract}
An approach is presented to solve the problem of spatial shift wrapping associated with spatial shift estimation-based fringe pattern profilometry (FPP). This problem arises as the result of fringe reuses (that is, use of fringes with periodic light intensity variance), and the spatial shift can only be identified without ambiguity within the range of a fringe width. It is demonstrated that the problem is similar to the phase unwrapping problem associated with the phase-detection-based FPP, and the proposed method is inspired by the existing ideas of using multiple images with different wavelengths proposed for phase unwrapping. The effectiveness of the proposed method is verified by comparing experimental results against several objects, with the last object consisting of more complex surface features. We conclude by showing that our method is successful in reconstructing the fine details of the more complex object. C 2014 SPIE and IS\&T [DOI: 10.1117/1.JEI.23.4.043002]
\end{abstract}

Keywords: fringe pattern profilometry; 3-D measurement; phase unwrapping.

Paper 14165P received Mar. 30, 2014; revised manuscript received Jun. 6, 2014; accepted for publication Jun. 6, 2014; published online Jul. 3, 2014.

\section{Introduction}

Fringe pattern profilometry (FPP) based on digital fringe projection (DFP) is a promising optical noncontact threedimensional (3-D) profile measurement technology. Compared to other methods, it has the advantages of a simple system structure and high accuracy. Figure 1 shows the structure of a DFP-based FPP, consisting of a digital projector, a camera, and a reference plane. With the system, a frame of image with a particular fringe pattern is produced by the digital projector and projected onto the reference plane, and then onto the surface of the object when the reference plane is removed. The projected images from the reference plane and the object surface are captured by a charge coupled device (CCD) camera, with the latter being a deformed version of the former because of the variance of the height of the object surface. As the deformed fringe pattern carries the information of surface shape, the 3-D profile of the object can be retrieved from these two fringe patterns.

Several FPP approaches have been developed during the past decades. The most widely used are those based on phase difference estimation (PDE). ${ }^{1}$ In these approaches, the projected fringe patterns are sinusoidal or periodic, and the deformed ones reflected from the object surface are considered the result of phase modulation of the original fringe pattern. The surface profile is obtained by detecting the phase maps of the two fringe patterns. A number of fringe pattern analysis methods have been developed, such as Fourier transform profilometry (FTP), ${ }^{2}$ phase shifting profilometry (PSP), ${ }^{3-5}$ modulation measurement profilometry, ${ }^{6}$ spatial phase detection, ${ }^{7,8}$ phase lock loop profilometry, ${ }^{9}$ Moire technique ${ }^{10}$ laser triangulation measurement, ${ }^{11}$ color-coded fringe projection, ${ }^{12-14}$ and other methods. ${ }^{15,16}$ Among these existing approaches, FTP and PSP are the most popular and widely used. ${ }^{1}$

*Address all correspondence to: Jiangtao Xi, E-mail: jiangtao@uow.edu.au
Although phase-based approaches are popular, they suffer from a number of weaknesses. A major restriction is that fringe patterns must be either sinusoidal or ideal periodic. However, such a requirement is hard to meet in practice due to factors such as the nonlinear distortion inherent to digital video projections. In order to solve the problem, a profilometry approach was proposed by $\mathrm{Hu}$ et al., ${ }^{17-19}$ which, rather than relying on detecting the differences between the phase maps, is based instead on the estimation of spatial shift for corresponding pixels on the two fringe patterns. The approach is referred to as the spatial shift estimation (SSE) profilometry approach. This approach has a great advantage. The projected fringe patterns are no longer required to be sinusoidal or periodic, leading to an increased freedom for the selection of the fringe patterns. Although the spatial shift estimation approach is suitable for any fringe pattern, use of periodic fringe patterns is still a straightforward choice. In the SSE-based approaches, the spatial shift between corresponding pixels on the two fringe patterns can only be detected within the range of $[0, \lambda]$, where $\lambda$ is the wavelength, or the spatial width of the individual fringe, i.e., number of pixels per fringe stripe. Obviously, shift unwrapping is also required in order to correctly restore the 3-D shape of the object surface. ${ }^{20}$ However, spatial shift unwrapping for a complex object using spatial shift estimation-based fringe pattern profilometry is still an outstanding issue. This problem has motivated the work presented in this paper.

The spatial shift unwrapping problem is similar to the phase unwrapping problem in phase-based FPP. The phase unwrapping problem arises because the phase can only be detected within the principle value range of $[-\pi, \pi]$, but the true phase can exceed the range. In order to retrieve the actual surface shape of the object, phase unwrapping must be carried out to obtain the actual phase maps. To solve the unwrapping problem, Zhang et al. ${ }^{21}$ introduced a multiple 
wavelength phase unwrapping algorithm. Using the method in Ref. 21, an image with a single fringe covering the whole measurement area is first projected, followed by a series of fringe images with a number of fringes for each image increasing by a constant factor. Since the first image only contains one fringe, unwrapping is not required. Then, the phase of the second image can be unwrapped by referring to the first image. After the phase of second image is obtained, it can be used to correct the third image, etc.

Inspired by the method, ${ }^{21}$ we introduce an approach to unwrap the spatial shift. In the proposed method, a series of fringe images are projected. The first image only has one fringe covering the whole measurement area, and the remaining ones are characterized by their wavelengths, which decrease by a constant factor. Similarly to Ref. 21 , the spatial shift unwrapping is sequentially carried out, starting from the images with long wavelengths to images with short wavelengths.

This paper is organized as follows. We begin in Sec. 2 by giving a brief introduction on conventional phase difference estimation-based fringe pattern profilometry and the spatial shift estimation-based technique, including their principles, system structures, and relevant algorithms. Then, in Sec. 3 we indicate that the unwrapping problem also exists in the spatial shift estimation approach, which is similar to the phase unwrapping problem in phase difference estimationbased fringe pattern profilometry. The paper then gives a review of the multiple-wavelength phase unwrapping algorithm introduced in Ref. 21, based on which we introduce a multiple-wavelength unwrapping algorithm for a spatial shift estimation approach. Finally, in Sec. 4, experimental results are given to demonstrate that the proposed method can be used to measure complex objects with a significant step height using the spatial shift estimation approach.

\section{Principle of Fringe Pattern Profilometry}

\subsection{Principle of Triangulation}

FPP is based on the triangulation principle described as follows. Without loss of generality and for simplicity in expression, we assume that the projector has a fringe structure. We can assume that light intensity varies periodically along the $x$ direction, while remaining constant along the $y$ direction, as shown in Fig. 1. We use $s(x), d(x)$, and $h(x)$ to denote the variance of light intensity of the fringe pattern

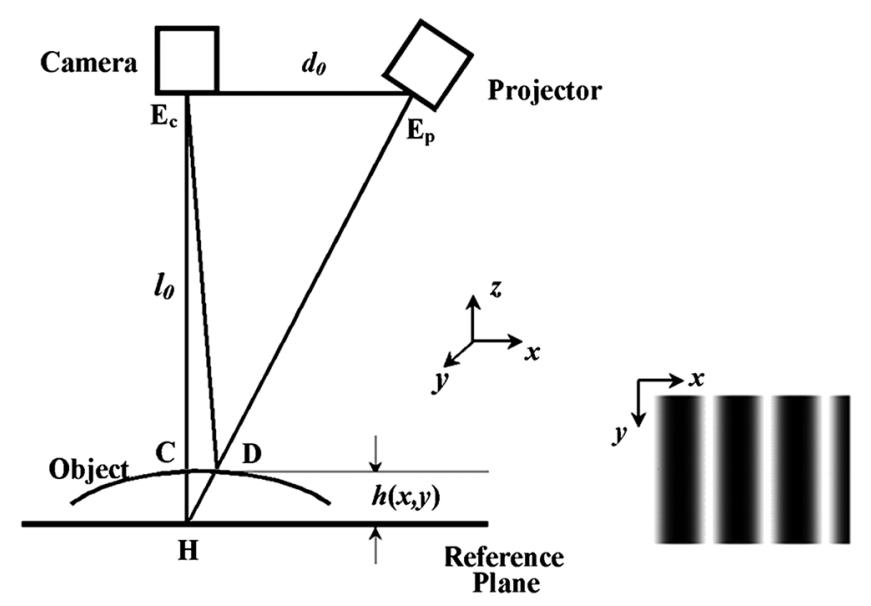

Fig. 1 Schematic diagram of FPP system. on the reference plane, the object surface, 1 and the height distribution along the $x$ coordinate, respectively. We also assume that the reference plane and the object surface have the same reflective characteristics.

Let us consider what happens when a beam of light is projected onto point $D$ on the object. When the object is removed, the same light beam (hence with the same intensity) should be projected onto point $H$ on the reference surface, which is reflected back to the camera through point $C$. As the triangles $E_{c} E_{P} H$ and $C D H$ are similar, we have the following relationship

$\frac{d_{0}}{l_{0}}=\frac{\overline{C D}}{h\left(x_{d}\right)}$.

Note that $x_{d}$ denotes the coordinate positions of point $D$. $h\left(x_{d}\right)$ denotes the distance between points $C$ and the reference plane, given by

$h\left(x_{d}\right)=\frac{l_{0} \overline{C D}}{d_{0}}$.

The above relationship gives the foundation for FPP.

\subsection{Phase Difference Estimation-Based Approaches for Fringe Pattern Profilometry}

The PDE-based FPP utilizes fringe patterns that are periodic and can be expressed as ${ }^{22,23}$

$s(x)=\sum_{k=0}^{+\infty} b_{k} \cos \left(2 \pi k f_{0} x+\psi_{k}\right)$,

and the deformed fringe pattern can also be expressed as

$d(x)=\sum_{k=0}^{+\infty} b_{k} \cos \left(2 \pi k f_{0} x+\phi_{k}(x)+\psi_{k}\right)$.

In the above equations, $f_{0}$ is the spatial frequency of the fringe patterns, and $b_{k}$ is the amplitude of the $k^{\prime}$ th order harmonic component. $\psi_{k}$ is the initial phase of the $k^{\prime}$ th order harmonic component, and $\phi_{k}(x)$ denotes the phase difference between the $k$ 'th order harmonic components of these two fringe patterns.

Equations (3) and (4) show that $s(x)$ and $d(x)$ are related by the phase shift $\phi_{k}(x)$. Let us consider the light beam projected at point $D$ on the object and $H$ on the reference plane when the object is removed. The phase shift between $C$ and $D$ can be determined by the spatial distance $\overline{C D}$, and hence we have $\mathrm{e}^{22,23}$

$\phi_{k}\left(x_{d}\right)=2 \pi k f_{0} \overline{C D}=k \cdot 2 \pi f_{0} \overline{C D}=k \cdot \phi\left(x_{d}\right)$,

where $\phi\left(x_{d}\right)=2 \pi f_{0} \overline{C D}$ is the phase shift of the fundamental component.

Substituting Eq. (5) into Eq. (2) we have

$h\left(x_{d}\right)=\frac{l_{0} \phi\left(x_{d}\right)}{2 \pi f_{0} d_{0}}$.

As points $D$ and $H$ are arbitrary, the derivations should apply to all the points on the projected fringe pattern. Therefore, we have 
$h(x)=\frac{l_{0} \phi(x)}{2 \pi f_{0} d_{0}}$.

Equation (7) shows that as long as $\phi(x)$ can be detected, we are able to calculate the height distribution $h(x)$ of the object surface. This is the foundation of all PDE-based approaches.

\subsection{Spatial Shift Estimation-Based Fringe Pattern Profilometry}

The PDE-based FPP methods suffer from some limitations. In particular, for the phase maps of $s(x)$ and $d(x)$ to exist and be detectable, the fringe patterns used for projection are limited to those which are sinusoidal or purely periodic. However, due to many undesired factors inherent to digital projection, such as geometrical distortion and nonlinear intensity distortion, purely sinusoidal fringe patterns are hard to produce. In order to solve these problems, Hu et al. ${ }^{17}$ introduced a method which is based on the SSE rather than PDE.

The SSE-based approach is rather simple and straightforward. Let us consider again $\overline{C D}$, where the distance between $C$ and $D$ is a function of the location of $D$ (i.e., $x_{d}$ ), the location of $H$ (or $C$, i.e., $x_{c}$ ), and the height of the object at point $H$, i.e, $h\left(x_{d}\right)$. Therefore, we have the following:

$\frac{d_{0}}{l_{0}}=\frac{u\left(x_{d}\right)}{h\left(x_{d}\right)}$,

where $u\left(x_{d}\right)=\overline{C D}=x_{c}-x_{d}$, which is the spatial distance between $x_{d}$ and $x_{c}$. Note that $x_{d}$ and $x_{c}$ are the points on $d(x)$ and $s(x)$ having the same light intensity, that is $d\left(x_{d}\right)=$ $s\left(x_{c}\right)$. As the above derivation is valid for any $x_{d}$ and $x_{c}$, we can replace $x_{d}$ by $x$, yielding the following:

$h(x)=\frac{l_{0} u(x)}{d_{0}}$.

Note that $u(x)$ is the spatial distance between point $x$ on $d(x)$ and the corresponding point on $s(x)$ with the same light intensity, that is

$d(x)=s[x-u(x)]$.

Equations (9) and (10) provide a straightforward way to obtain the 3-D profile of the object surface. With $d(x)$ and $s(x)$ available, if we are able to obtain $u(x)$ to meet Eq. (10), we then can utilize Eq. (9) to yield $h(x)$, the height distribution of the object surface along $x$. By repeating the procedure for all $y$, we should be able to obtain the 3-D profile of the object surface.

A number of approaches were proposed to retrieve the $u(x) \cdot{ }^{17-19}$ Among these approaches, the one referred to as inverse function-based shift estimation (IFSE) ${ }^{19}$ is particularly interesting. Wu et al. ${ }^{24}$ improved this method by combining IFSE with the multiple-step triangular-pattern phase shifting algorithm, ${ }^{25}$ which greatly improved the accuracy of the measurement.

The spatial shift-based approach has a particular advantage. The projected fringe patterns are no longer required to be sinusoidal, which implies that even though there are distortions within the fringe patterns, sufficient 3-D information on the object surface is contained in the variations between projected and deformed fringe patterns. Thus profilometry can be achieved.

\section{Unwrapping Problem}

With most PDE-based approaches, the phase is obtained by an operation involving the arctangent function, and thus the retrieved phase value $\phi(x)$ can only be identified within the range of $[-\pi, \pi]$. In other words, $\phi(x)$ is obtained by the modulo $2 \pi$ operation, resulting in discontinuities in its values near $\pi$ ( $\pi$ and $-\pi$ ) appearing in the phase map. This is called the wrapping problem. In order to recover the absolute phase $\Phi(x)$ from the wrapped value, we have

$\Phi(x)=2 \pi m(x)+\phi(x)$,

where $m(x)$ is an integer indicating the number of $2 \pi$ lost due to the wrapping problem, and the procedure of retrieving $m(x)$ is called phase unwrapping. ${ }^{21}$

\subsection{Spatial Shift Unwrapping in Spatial Shift Estimation-Based Fringe Pattern Profilometry}

The wrapping problem also exists in SSE approaches. From Eq. (9) we have

$u(x)=\frac{d_{0} h(x)}{l_{0}}$.

Depending on $h(x), d_{0}$, and $l_{0}$, the shift function $u(x)$ may take any value as well. However, when $s(x)$ has a fringe structure with a periodic fringe of width $\lambda, u(x)$ can only be detected within the main value of $[0, \lambda]$. In other words, $u(x)$ is wrapped into $[0, \lambda]$. Thus, the true shift function, denoted as $U(x)$, should be continued and recovered from $u(x)$ as follows:

$U(x)=\lambda m(x)+u(x), \quad$ where $m(x)$ are integers.

In order to demonstrate the relationship, we utilize the example in Fig. 2. With $h(x)$ shown in Fig. 2(a), we should have $U(x)$ in Fig. 2(b). However, due to the wrapping problem, what we do have is the wrapped version of the shift $u(x)$ in Fig. 2(c). If $u(x)$ is used in Eq. (9) without unwrapping, a significant error will occur in the construction of $h(x)$, as shown by Fig. 2(d). Therefore, we must work out a way to restore $U(x)$. This restoration process is referred to as spatial shift unwrapping.

\subsection{Multiple-Wavelength Phase Unwrapping Algorithm}

To solve the phase unwrapping problem, Zhang et al. ${ }^{21}$ introduced a multiple-wavelength phase unwrapping algorithm. With the method proposed in Ref. 21, a series of fringe patterns are projected on a one-by-one basis. Suppose the measurement area has a resolution of $W \times H$ and the projected fringe images are vertical, the first fringe pattern only has a single fringe with its spatial wavelength $\lambda_{1}$ the same as $W$. As the fringe covers the whole measurement area, phase unwrapping is not required and hence $\phi_{1}(x)=$ $\Phi_{1}(x)$ and $m_{1}(x) \equiv 0$.

After the first fringe pattern, a series of fringe images are then projected with their wavelength decreased by a factor $1 / N$. In other words, the wavelength of the $k$ 'th fringe pattern given by $\lambda_{k}=N \lambda_{k+1}$ is used. As we know, 


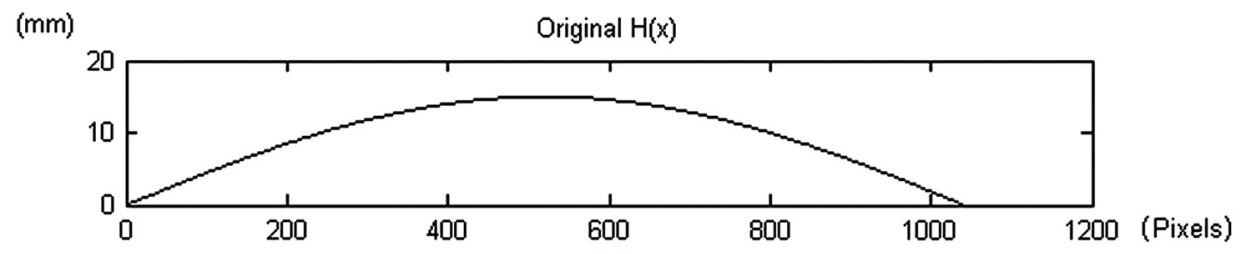

(a)

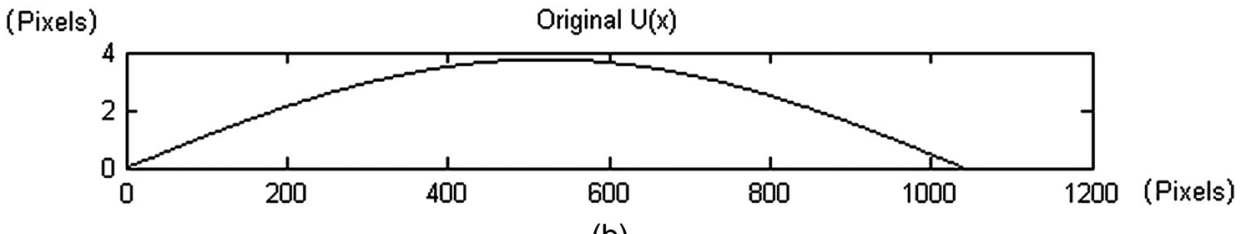

(b)

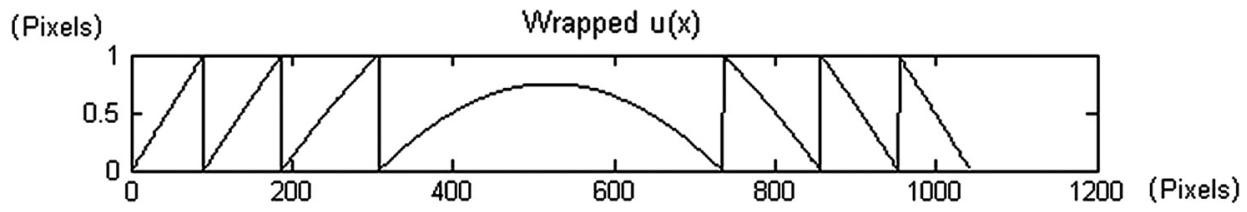

(c)

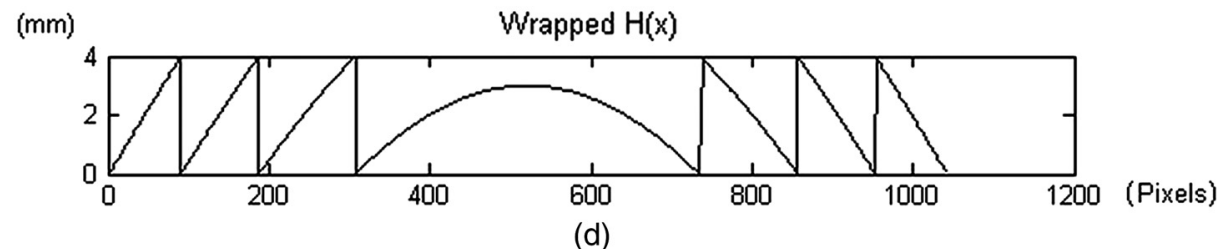

Fig. 2 Unwrapped and wrapped shift maps. (a) original $H(x)$; (b) original $U(x)$; (c) wrapped $u(x)$; (d) wrapped $H(x)$.

$\phi_{1}(x)=\Phi_{1}(x)$, and since $\lambda_{1}=N \lambda_{2}$, we will have $\Phi_{2}(x)=$ $N \Phi_{1}(x) .{ }^{21}$ Combining this with Eq. (11) yields the following:

$m_{2}(x)=$ Integer $\left[\frac{N \Phi_{1}(x)}{2 \pi}-\frac{\phi_{2}(x)}{2 \pi}\right]$,

where the operator integer [] obtains the closest integer value. The wrapped phase, $\phi_{2}(x)$, can be unwrapped on pixel-bypixel basis by referring to the retrieved $m_{2}(x)$

$\Phi_{2}(x)=2 \pi m_{2}(x)+\phi_{2}(x)$.

After $\Phi_{2}(x)$ is obtained, it can be used to recover $\phi_{3}(x)$. Therefore, in general, for $\lambda_{k}=\lambda_{k-1} / N$,

$m_{k}(x)=$ Integer $\left[\frac{N \Phi_{k-1}(x)}{2 \pi}-\frac{\phi_{k}(x)}{2 \pi}\right]$,

and the absolute phase map $\Phi_{k}(x)$ can be retrieved using Eq. (11).

Since the unwrapped phase is obtained without relying on pixel neighborhoods within the same phase map, unwrapping errors will not propagate from one noisy neighborhood to spatially adjacent regions of the image. Similarly, noise contained in images obtained using longer wavelengths will not propagate into images obtained using shorter wavelengths. Therefore, this technique can more accurately measure complex surfaces which may contain significantly elevated profile steps, while at the same time improving reliability and noise immunity by minimizing the propagation of localized phase unwrapping errors.

\subsection{Multiple-Wavelength Spatial Shift Unwrapping Algorithm}

In order to work out how to unwrap the spatial shift, a simple spatial shift unwrapping method had been proposed in Ref. 20 which unwraps the spatial shift using the neighborhood pixels. However, this method cannot retrieve the correct shift distribution if the value difference of the neighborhood pixels is greater than $\lambda / 2$. Hence, it cannot solve the unwrapping problem for those complex objects.

To address this problem, we introduce a multiple-wavelength unwrapping algorithm for a spatial shift estimationbased approach based on Zhang's method. ${ }^{21}$

In this algorithm, we still assume that the measurement area has a resolution of $W \times H$ and the projected fringe images are vertical. Then, the wavelength of the fringe which is projected initially, is selected to be $W$, that is $\lambda_{1}=W$. Since this single fringe stripe covers the whole measurement area, spatial shift unwrapping is not needed. Hence, we have $u_{1}(x)=U_{1}(x)$ and $m_{1}(x) \equiv 0$.

We, then choose $\lambda_{2}=\lambda_{1} / N$. From Eq. (12), it is easy to see that the real shift function $U(x)$ is only related to the height distribution $h(x)$ and two distances $d_{0}$ and $l_{0}$. Since these values are fixed, the true shift functions for different wavelengths $\lambda$ should be the same, that is, $U_{2}(x)=U_{1}(x)=$ $U(x)$. Thus, combining this equation with Eq. (13), we have

$m_{2}(x)=$ Integer $\left[\frac{U_{1}(x)}{\lambda_{2}}-\frac{u_{2}(x)}{\lambda_{2}}\right]$. 


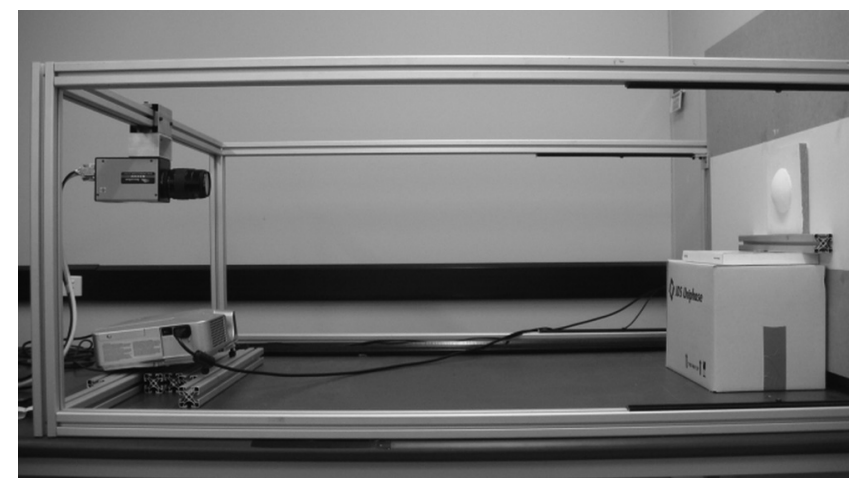

Fig. 3 The experimental system setup.

This means that $u_{2}(x)$ can now be unwrapped using $m_{2}(x)$, which in turn was obtained based on information contained in the longer wavelength shift $U_{1}(x)$. This can be carried out on a pixel-by-pixel basis

$U_{2}(x)=\lambda_{2} m_{2}(x)+u_{2}(x)$.

Because shift $U_{1}(x)$ is only used for obtaining the integer $m_{2}(x)$, the noise of $U_{1}(x)$ does not propagate to $U_{2}(x)$. With $U_{2}(x)$ retrieved, now we can use it to obtain $U_{3}(x)$, where $\lambda_{3}=\lambda_{2} / N$. Therefore, in general, for $\lambda_{k}=\lambda_{k-1} / N$, we have

$m_{k}(x)=$ Integer $\left[\frac{U_{k-1}(x)}{\lambda_{k}}-\frac{u_{k}(x)}{\lambda_{k}}\right]$,

and the real shift function $U(x)$ can be retrieved using Eq. (13).

From Eq. (19), we can find that the unwrapped spatial shift map is retrieved by referring to the longer wavelength shift map. Compared to algorithms which use neighborhood pixels to do the unwrapping, ${ }^{20}$ the method we propose here can measure the surface profile with larger steps. This is because the noise of longer wavelengths' images will not propagate to the shorter ones, therefore, the measurement's overall accuracy is purely determined by the image with the shortest wavelength.

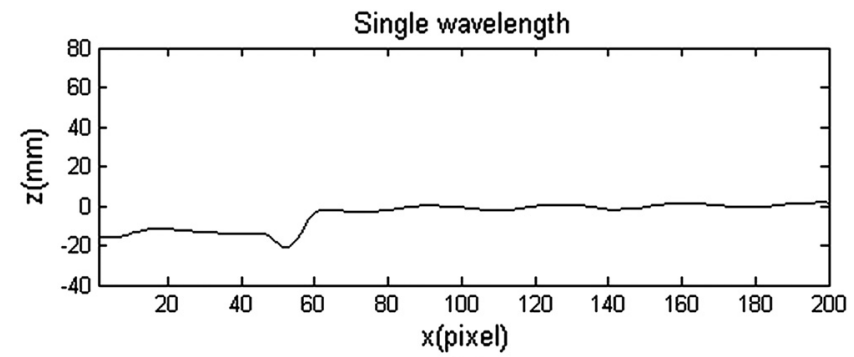

(a)

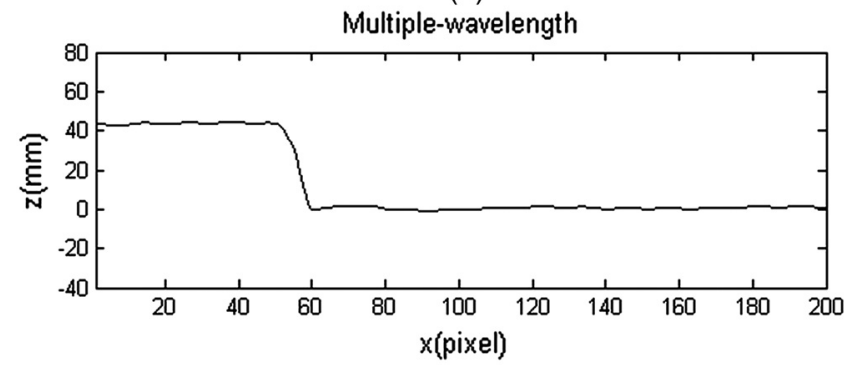

(b)

Fig. 5 Cross section of flat box. (a) Result using single wavelength algorithm; (b) result using multiple-wavelength algorithm.

\section{Experiments and Results}

In order to test the performance of the approach proposed in Sec. 3, experiments were carried out in our laboratory. The experimental setup is shown in Fig. 3. The triangular fringe patterns $^{24}$ are projected by a Hitachi Ltd., Chiyoda, Tokyo, Japan CP-X260, and a Duncan Technologies Inc., California, MS3100 3 CCD digital camera is used to capture the fringe patterns. The digital camera is placed on top of the projector with a distance of $350 \mathrm{~mm}$. The distance between the camera lens and the reference plane is $1295 \mathrm{~mm}$. The resolution of the CCD camera is $1392 \times 1039$ pixels, and the field of vision for the CCD camera is $250 \times 187 \mathrm{~mm}$. Hence, the equivalent spatial resolution is $0.1796 \mathrm{~mm} /$ pixel.

To verify that the proposed method can measure the surface profile with an arbitrary step height, we first chose a flat box with a height of $43 \mathrm{~mm}$ as the measured object. Figure 4(a) shows the photograph of the object. Figures 4(b)-4(d) show the captured three-step triangular patterns fringe images of

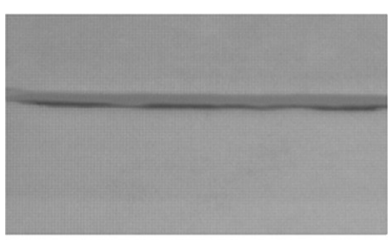

(a)

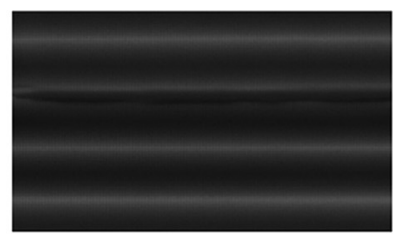

(b)

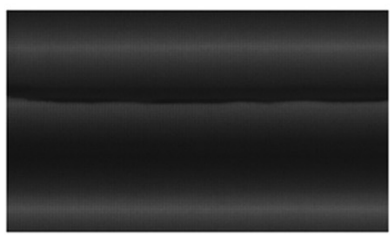

(e)

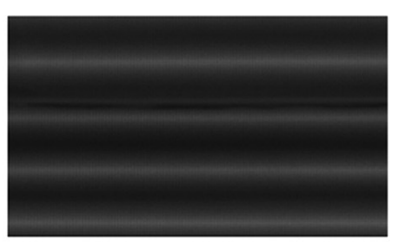

(c)

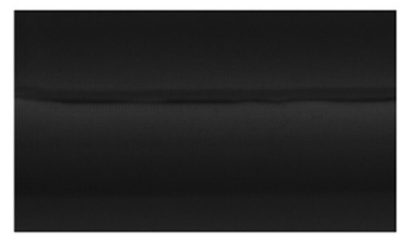

(f)

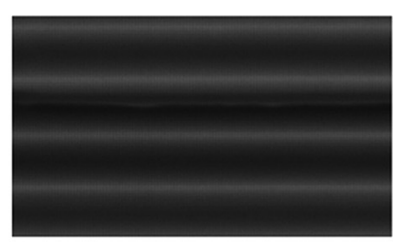

(d)

Fig. 4 Captured fringe images of flat box. (a) object; (b)-(d) fringes images $(\lambda=20)$; (e) fringes image $(\lambda=40)$; (f) fringes image $(\lambda=80)$. 


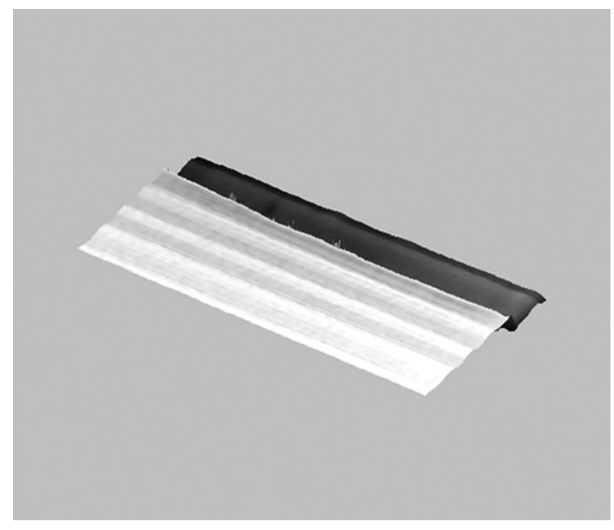

(a)

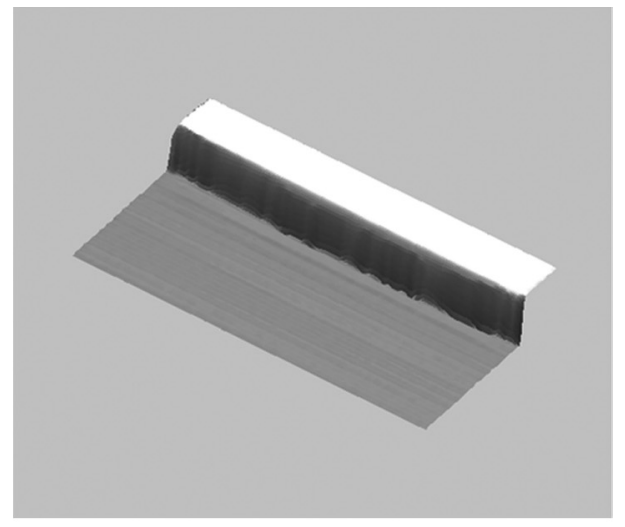

(b)

Fig. 6 3-D reconstruct results of flat box. (a) result using single wavelength algorithm, (b) result using multiple-wavelength algorithm.

the object with wavelength $\lambda=20$. Figures 4(e) and 4(f) show the captured fringe images of the object with wavelength $\lambda=40$ and $\lambda=80$.

We first reconstruct this object using a single wavelength triangular-pattern spatial-shifting algorithm. The wrapped $u(x)$ is retrieved using the method in Ref. 24, and it is unwrapped using the method in Ref. 20. In contrast, the multiple-wavelength algorithm proposed is also used. Figure 5 shows the cross section of the object and Fig. 6 shows the $3-\mathrm{D}$ reconstructed results.

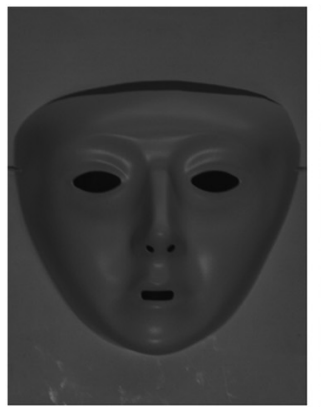

(a)

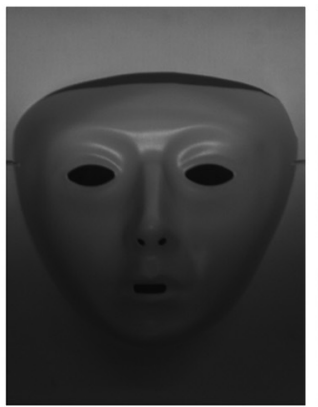

(b)

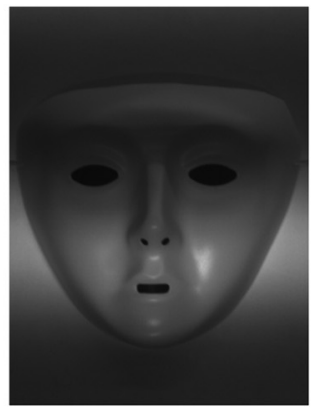

(c)

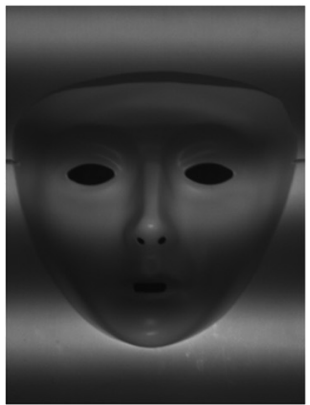

(d)

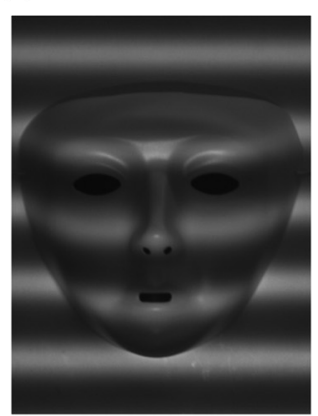

(e)

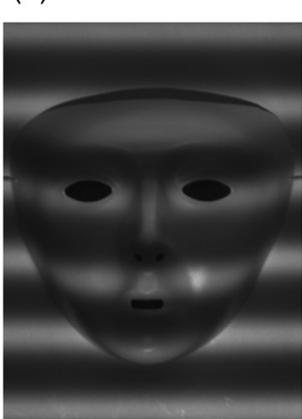

(h)

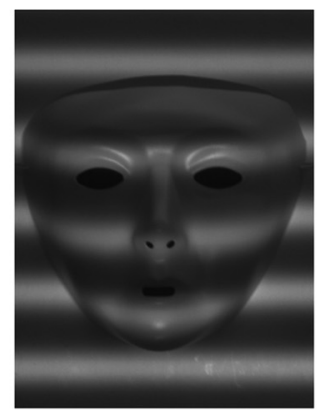

(f)

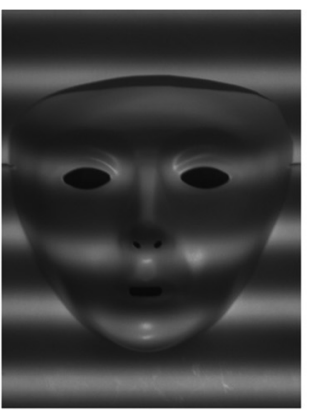

(g)

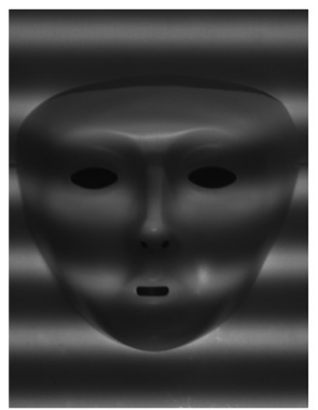

(i)

Fig. 7 Captured fringe images of mask with different wavelengths. (a) object, (b) fringes image $(\lambda=480)$, (c) fringes image $(\lambda=240)$, (d) fringes image $(\lambda=120)$, (e)-(i) fringes images $(\lambda=60)$. 


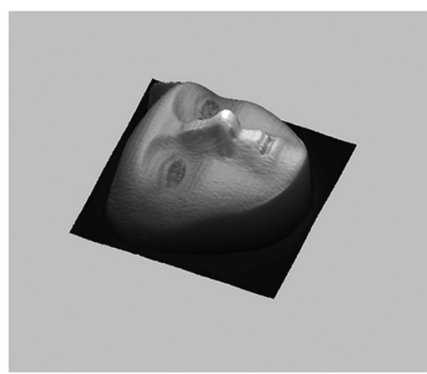

(a)

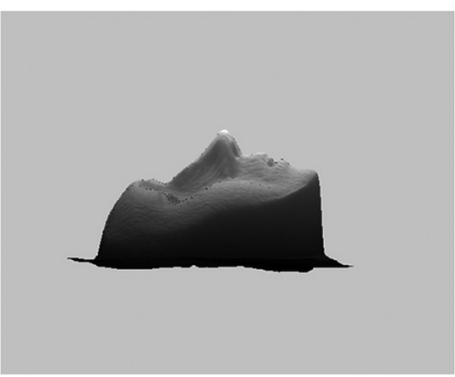

(b)

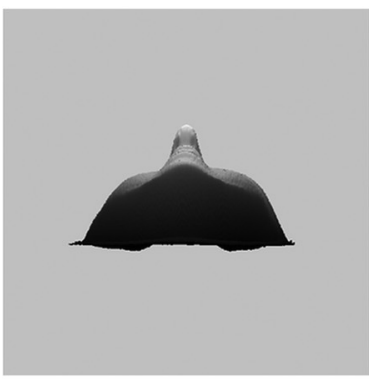

(c)

Fig. 8 3-D reconstruct results of mask. (a)-(c) Results in different angle of view.

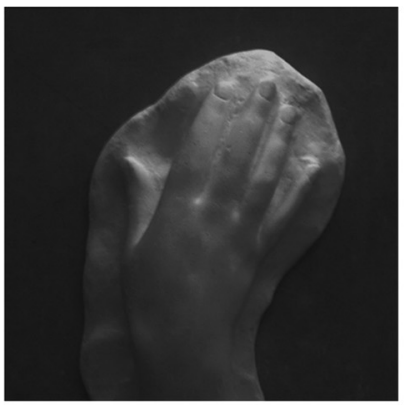

(a)

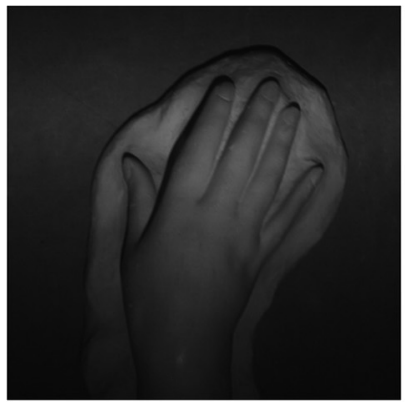

(b)

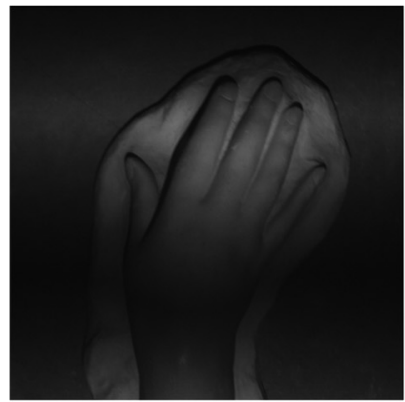

(c)

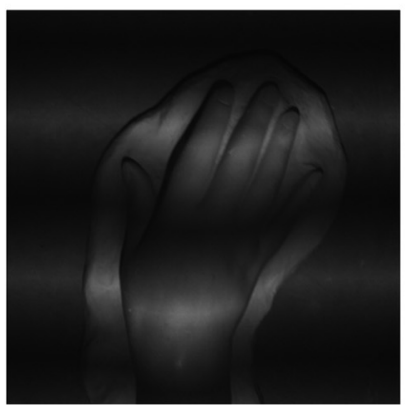

(d)

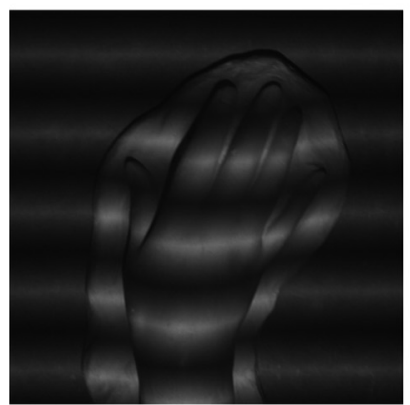

(e)

Fig. 9 Captured fringe images of hand model with different wavelengths. (a) object; (b) fringes image $(\lambda=480)$; (c) fringes image $(\lambda=240)$; (d) fringes image $(\lambda=120) ;(e)$ fringes image $(\lambda=60)$.

It can be seen from Figs. 5 and 6 that the result using a single wavelength algorithm is not correct; it generates a box height result of about $-17 \mathrm{~mm}$. On the other side, using the multiple-wavelength algorithm, the step height can be measured successfully; the height result is about $43 \mathrm{~mm}$. This experiment demonstrated that the proposed approach can successfully perform the measurement even when the surface profile has a step.

We then test the performance of the proposed method for the measurement of a mask characterized by a complex surface shape. In this experiment, a four-wavelength multiple-step triangular-pattern spatial-shifting algorithm with

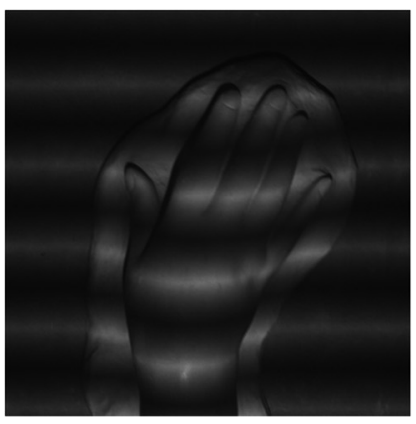

(a)

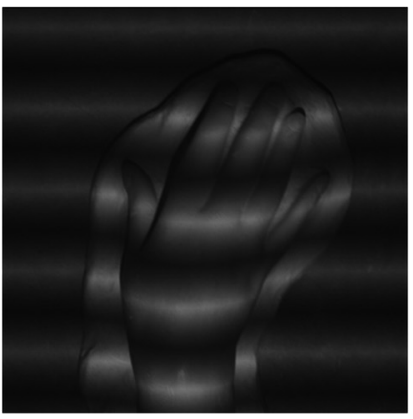

(b)

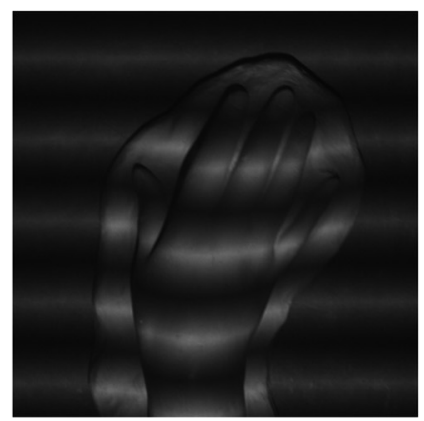

(c)

Fig. 10 Captured three-step fringe images of hand model with wavelength $\lambda=60$. (a)-(c) Fringe images in different step. 


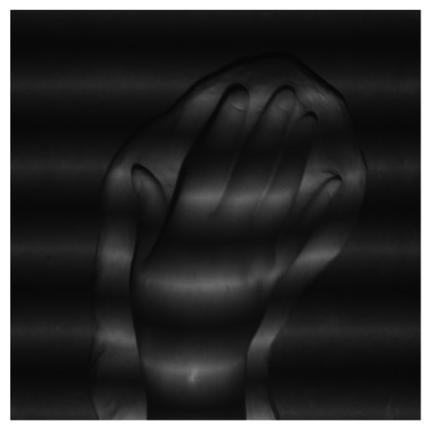

(a)

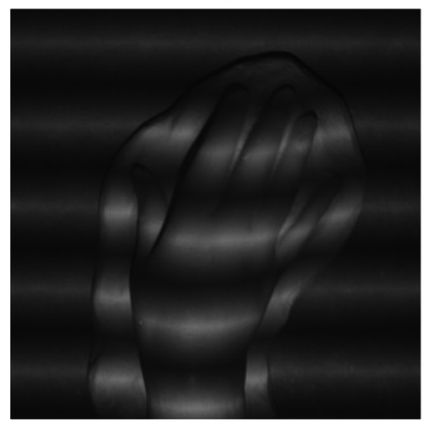

(d)

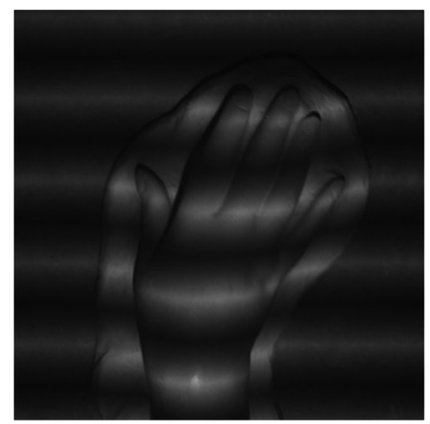

(b)

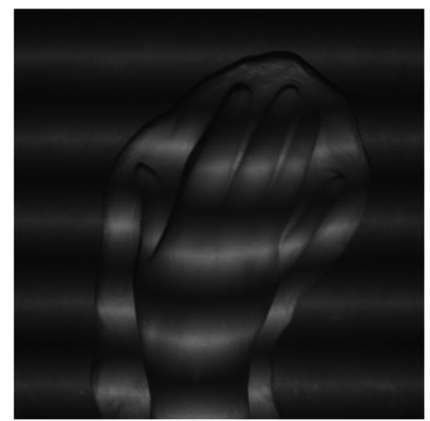

(e)

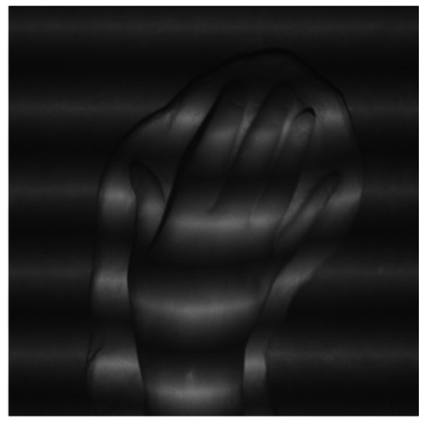

(c)

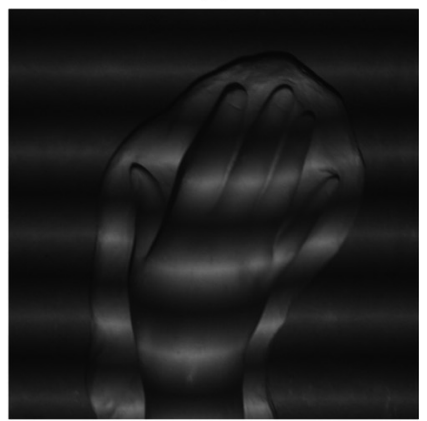

(f)

Fig. 11 Captured six-step fringe images of hand model with wavelength $\lambda=60$. (a)-(f) Fringe images in different step.

$\lambda_{1}=480$ and $N=2$ is used. Figure 7(a) shows the mask and Figs. 7(b)-7(d) show the captured fringe images of the object with wavelengths $\lambda_{1}=480, \lambda_{2}=240$, and $\lambda_{3}=120$. For the shortest wavelength $\lambda_{4}=60$, five-step triangular patterns are used to construct the 3-D shape. Figures 7(e)-7(i) show the captured five fringe images of the object.

Figure 8 shows the reconstructed 3-D surface shape of the object using the proposed unwrapping method. It can be seen from these figures that the mask is reconstructed successfully. The height information for those places with a high step drop (mask edge and nose part) is correctly retrieved. Some details, such as mouth and eyes parts, are also recovered. This result demonstrated that the proposed method can successfully measure those complex objects with an arbitrary step height.

We also tested the proposed technique using another complex object which is a plaster hand model. As mentioned in Sec. 3.3, the noise of images with longer wavelengths will

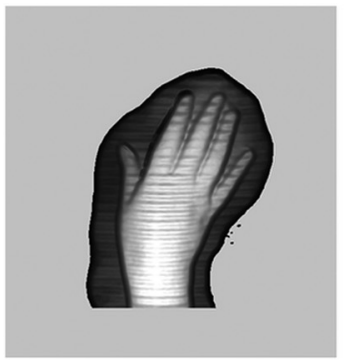

(a)

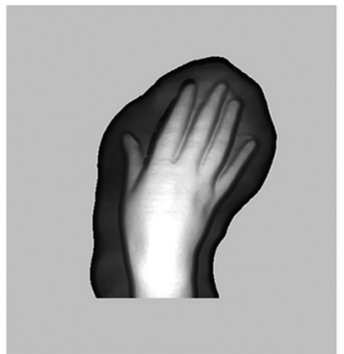

(e)

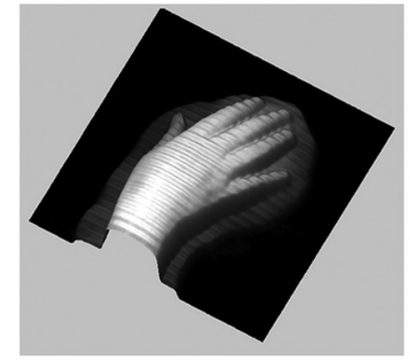

(b)

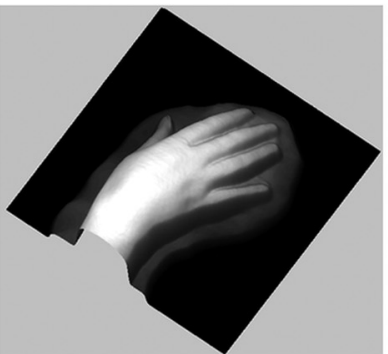

(f)

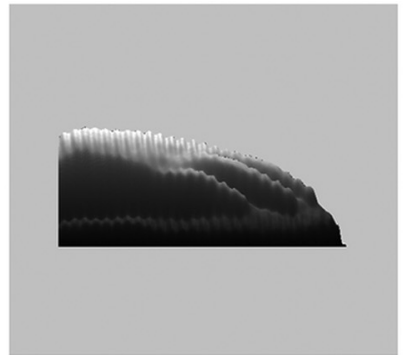

(c)

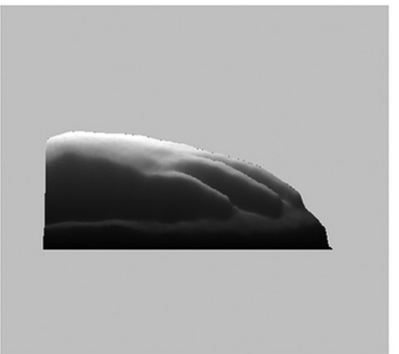

(g)

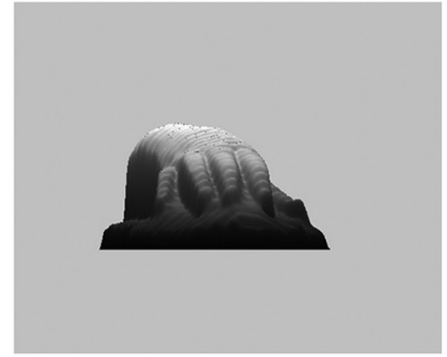

(d)

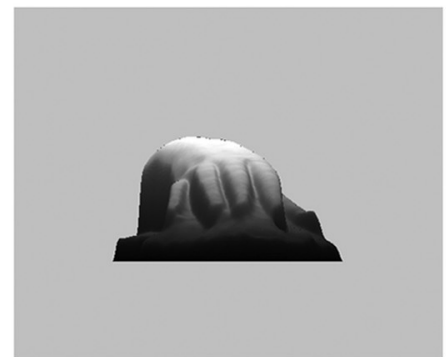

(h)

Fig. 12 3-D reconstruct results of hand model. (a)-(d) result using three-step fringe images; (e)-(h) result using six-step fringe images. 
not propagate to the shorter ones, and the overall accuracy is purely determined by the image with the shortest wavelength. Hence, in this experiment, we performed two measurements with a different number of fringe images using the shortest wavelength. The number of images used in the longer wavelengths is kept the same. Figure 9(a) shows the model and Figs. 9(b)-9(e) show the captured fringe images of the object with different wavelengths. Figure 10 shows the captured three-step fringe images of the hand model with wavelength $\lambda=60$. Six-step fringe images of the hand model with wavelength $\lambda=60$ is shown in Fig. 11 .

Figure 12 depicts the reconstructed 3-D surface shape of the hand model using the proposed unwrapping method, where Figs. 12(a)-12(d) show the results using three-step fringe images and Figs. 12(e)-12(h) show the results using six-step fringe images. As shown in these figures, the model is successfully reconstructed in both measurements. Besides, it is noticed that the quality of the reconstructed model is also improved with an increase in the number of images with the shortest wavelength. The results confirmed that noise does not propagate from long wavelength patterns to short ones. Therefore, by means of increasing the number of images with the shortest wavelength, we can achieve a more accurate measurement of complex surface shapes.

\section{Conclusion}

In this paper, we studied the spatial shift wrapping problem associated with SSE-based FPP.

Compared to PDE-based approaches, SSE techniques are advantageous in that nonsinusoidal fringe patterns can be employed, and they do not suffer from the nonlinear distortion associated with the digital fringe projection. However, similar to the phase unwrapping problem encountered in PDE-based approaches, a shift unwrapping problem also exists in SSEbased approaches. While SSE-based profilometry was proposed a few years ago, there is not a work devoted to unwrap the spatial shift map for complex objects, and hence we presented a technique to carry out spatial shift unwrapping to overcome this limitation. In particular, we applied the method proposed in Ref. 21 for unwrapping the spatial shift. In order to test the performance of our proposed method, we carried out experiments on three objects which have arbitrary step heights and complex surface shapes: a flat box, a mask, and a plaster hand model. The results show that the proposed method can be used for surfaces with sudden height changes.

It should be pointed out that the number of image patterns required in this method is still an issue. For example, where the image pattern has 16 fringes, at least five image patterns are still required with this approach. As a future work, we will investigate the possibilities for reducing the number of image patterns necessary to capture an image while maintaining the measurement's accuracy.

\section{References}

1. S. Gorthi and P. Rastogi, "Fringe projection techniques: whither we are?," Opt. Lasers Eng. 48(2), 133-140 (2010).

2. X. Su and W. Chen, "Fourier transform profilometry: a review," Opt. Lasers Eng. 35(5), 263-284 (2001).

3. H. Zhang, M. J. Lalor, and D. R. Burton, "Spatiotemporal phase unwrapping for the measurement of discontinuous objects in dynamic fringe-projection phase-shifting profilometry," Appl. Opt. 38(16), 3534-3541 (1999).

4. M. Halioua and H. C. Liu, "Optical three-dimensional sensing by phase measuring profilometry," Opt. Lasers Eng. 11(3), 185-215 (1989).
5. Y. Fu and Q. Luo, "Fringe projection profilometry based on a novel phase shift method," Opt. Express 19(22), 21739-21747 (2011).

6. X. Su et al., "New 3D profilometry based on modulation measurement," Proc. SPIE 3853, 1-7 (1998).

7. S. Toyooka and M. Tominga, "Spatial fringe scanning for optical phase measurement," Opt. Commun. 51(2), 68-70 (1984).

8. S. Toyooka and Y. Iwaasa, "Automatic profilometry of 3-D diffuse objects by spatial phase detection," Appl. Opt. 25(10), 1630-1633 (1986).

9. R. Rodriguez-Vera and M. Servin, "Phase locked loop profilometry," Opt. Laser Technol. 26(6), 393-398 (1994).

10. D. M. Meadows, W. Johnson, and J. B. Allen, "Generation of surface contours by moiré patterns," Appl. Opt. 9(4), 942-947 (1970).

11. A. Asundi and Z. Wensen, "Unified calibration technique and its applications in optical triangular profilometry," Appl. Opt. 38(16), 3556-3561 (1999).

12. P. Huang et al., "Color-enhanced digital fringe projection technique for high-speed 3-D surface contouring," Opt. Eng. 38(6), 1065-1071 (1999).

13. Z. Zhang, C. E. Towers, and D. P. Towers, "Robust color and shape measurement of full color artifacts by RGB fringe projection," Opt. Eng. 51(2), 021109 (2012).

14. J. Guo et al., "Real-time 3D imaging by using color structured light based on Hilbert transform," Proc. SPIE 8856, 885624 (2013).

15. A. J. Moore and F. Mendoza-Santoyo, "Phase demodulation in the space domain without a fringe carrier," Opt. Lasers Eng. 23(5), 319-330 (1995)

16. J. Villa, M. Servin, and L. Castillo, "Profilometry for the measurement of 3-D object shapes based on regularized filters," Opt. Соттип. 161(1-3), 13-18 (1999)

17. Y. Hu et al., "Three-dimensional profilometry based on shift estimation of projected fringe patterns," Appl. Opt. 45(4), 678-687 (2006).

18. Y. Hu et al., "Discrete cosine transform based shift estimation for fringe pattern profilometry using generalized analysis model," Appl. Opt. 45(25), 6560-6567 (2006).

19. Y. Hu et al., "Inverse function analysis method for fringe pattern profilometry," IEEE Trans. Instrum. Meas. 58(9), 3305-3314 (2009).

20. P. Cao et al., "A fringe period unwrapping technique for digital fringe profilometry based on spatial shift estimation," Proc. SPIE 7432, 743208 (2009).

21. S. Zhang, "Phase unwrapping error reduction framework for a multiplewavelength phase-shifting algorithm," Opt. Eng. 48(10), 105601 (2009).

22. M. Takeda, H. Ina, and S. Kobayashi, "Fourier-transform method of fringe-pattern analysis for computer-based topography and interferometry," J. Opt. Soc. Am. 72(1), 156-160 (1982).

23. M. Takeda and K. Mutoh, "Fourier transform profilometry for the automatic measurement of 3-D object shapes," Appl. Opt. 22(24), 3977-3982 (1983).

24. K. Wu et al., "3D profile measurement based on estimation of spatial shifts between intensity ratios from multiple-step triangular patterns," Opt. Lasers Eng. 51(4), 440-445 (2013).

25. P. Jia, J. Kofman, and C. English, "Multiple-step triangular-pattern phase-shifting and the influence of number of steps and pitch on measurement accuracy," Appl. Opt. 46(16), 3253-3262 (2007).

$\mathrm{Pu}$ Cao is a $\mathrm{PhD}$ candidate at the University of Wollongong. He received his $\mathrm{BE}$ degree in electronic engineering from Huazhong University of Science and Technology, China, in 2006 and his ME degree in electrical engineering from the University of Wollongong, Australia, in 2010.

Jiangtao $\mathbf{X} i$ is a professor at the University of Wollongong. He received his BE degree from Beijing Institute of Technology, China, in 1982, his ME degree from TsingHua University, China, in 1985, and his $\mathrm{PhD}$ degree from the University of Wollongong, Australia, in 1996 respectively, all in electrical engineering. His current research interests include signal processing with applications to instrumentation and measurements, telecommunications, and optoelectronics systems. $\mathrm{He}$ is a senior member of IEEE and a member of SPIE.

Yanguang $\mathbf{Y u}$ is a senior lecturer at the University of Wollongong, Australia. She received her $\mathrm{BE}$ and $\mathrm{PhD}$ degrees, respectively, from Huazhong University of Science and Technology in 1986 and Harbin Institute of Technology in 2000 in China. Her current research interests include optoelectronic systems, 3-D profile measurement, and telecommunication systems.

Qinghua Guo is a lecturer at the University of Wollongong. He received his $B E$ degree in electronic engineering, his $M E$ degree in signal and information processing from Xidian University, in 2001 and 2004, respectively, and his $\mathrm{PhD}$ degree in electronic engineering from the City University of Hong Kong in 2008. He was a recipient of the Australian Research Council's inaugural Discovery Early Career Researcher Award. 